\title{
Photon Emitting, Absorption and Reconstruction of Photons
}

\author{
Changjun Liao, Zhengjun Wei and Jindong Wang \\ University Laboratory of Guangdong, School for Information \\ and Optoelectronic Science and Engineering \\ South China Normal University, Guangzhou \\ China
}

\section{Introduction}

Photon cannot keep itself unchanged from emission to absorption. The information encoded on the photon is also changed due to interaction with environments. There has no definitely demonstration that the photon absorbed is the original one from ideal light source since the quantum mechanics itself is an indeterminate theory that the physical measurement is only the probability. Any operation on photon before detector involves unavoidably loss that means a quantum permutation with environments. Although a photon is detected with same energy the phase uncertainty exists. Section 2 describes the single photon sources and the questions about the conception of photons. The third section describes the quantization of electromagetic fields that makes the basis of the Fork states in that the number of the photon is considered. In Section 4, the representation of the photon in space is described by a complete set of eigenfunctions which represent the fundamental modes with different eigen values. Concept of optical modes is a result of quantization of electromagnetic fields. Optical modes can be occupied by photons in different way in comparison with levels in atomic system in which Coulomb interaction considered. Bunching and anti-bunching are considered as the fundamental properties of the optical modes in Section 5. Based on these theories, several applications are considered. Directional emission of single photons is considered in Section 6, study on single photon detectors is presented in Section 7, multipartite entanglement and its application in quantum key distribution is introduced in Section 8. The fluctuation in vacuum, dephase and decoherent are considered in Section 9. General consideration of reconstructions of photons including coherent combination and interfere coherently, resonant-enhanced density of photons are put in the last Section. Here in this chapter photons are considered as field quantum theory instead of that quantum field theory. Photons consist of mode fields which are quantized.

\section{Sources of single photons}

The concept of Photon has important contribution to the foundation of quantum mechanics which can be seen in any textbook of quantum mechanics (Greiner, 2001). The "particles" of light are called quanta of light or photons which are recognized to have wave-particle duality, a typical feature of a quantum system. The concept of photon was soon 
demonstrated by photoelectric effect. This effect was interpreted by Einstein with his famous formula

$$
E=\hbar\left(\omega-\omega_{a}\right)=h\left(v-v_{a}\right)
$$

where $\mathrm{E}$ is a discrete quanta of light with energy of $\hbar \omega$, the Planck's constant $h=2 \pi \hbar$. The quantized energy was soon demonstrated with many experiments, including the Compton effects, the Ritz Combination principle, the Franck-Hertz experiment, etc.

Photons are ideal for quantum information applications due to its high transmission speed and easy to be coded with quantum information.

Single photons and entangled photon pairs are important for quantum information. Single photons are usually used in quantum key distribution (QKD) system as quantum information carriers to ensure the security of a key distribution system based on quantum mechanics principles (Gisin et al., 2002). The quantum mechanics assured that a single photon can not be divided and a single photon can not be cloned either (Wootters \& Zurek, 1982). Furthermore, a single measurement is not enough for determining the quantum state with certainty, any measurement provides only the probability if the state is unknown and the original state changed after measurement and can never be recovered again. Therefore study on generation and detection of single photons is extremely important.

Light sources are everywhere. But real single photon sources are facing technical challenges. Among many methods to provide single photons, three kinds of single photon sources have attracted much attention: the faint optical pulses, spontaneous down conversion photon pairs and quantum dot.

\subsection{Faint laser pulses}

In practice, the single photons are usually produced by precisely controlled attenuation of laser pulses to a very weak level and assume at that level the photon follow Poisson distribution.

$$
P_{n}=\frac{\mu^{n}}{n !} e^{-\mu}
$$

where, $P_{n}$ are the probability of the pulse containing $n$ photons with mean photon number of $\mu$ (Walls \& Milburn, 1994). The probability of containing more than one photon in faint pulses can be made arbitrarily small. For example, with mean photon number of 0.1 as quite usual, there is only $5 \%$ of the nonempty pulses contain photons more than one, most of the nonempty pulses contain only single photon. These single photon sources are pseudosingle-photons or correctly called faint laser pulses.

\subsection{Down-conversion photon pairs}

Single photon generators using correlated photon pairs generated by the spontaneous parametric down-conversion are widely reported (Mason et al., 2002; Migdall et al., 2002; Pittman et al., 2002; Walton et al., 2001). In particular, a periodically poled lithium niobate waveguide has high probability for generation of photon pairs at $1550 \mathrm{~nm}$ (Tanzilli et al., 2001; Yoshizawa et al., 2003; Mori et al., 2004). A photon pairs includes a signal photon and an idler photon correlated in time. Therefore the detection of idler photon can be used to control an optical switch so that the signal photon can surely emit in time. However, the 
number distribution of the photon pairs follows also Poissonian statistics. A photon number resolving detector is needed for idler photon if single photon emitting should be guaranteed as required in quantum information applications. Photon number resolving detection faces also technical challenges although several kinds of photon number resolving detectors have been reported, they still far from commercially available (Kardyna et al., 2007; Miller et al., 2003).

\subsection{Single quantum dot emission}

In theoretical consideration, single photons should come from a single transition between two single state levels by one electron in single quantum cavity, such as single atom, quantum dot, etc. It might be properly called "turn style" or "photon gun".

A single photon generation using semiconductor quantum dot has been reported (Santori et al., 2001). Electrically driven single-photon source has been demonstrated experimentally (Zhiliang Yuan et al., 2002). In their experiments, at low injection currents, the dot electroluminescence spectrum reveals a single sharp line due to single exciton recombination (one electron and one hole within a quantum dot), while another line due to the biexciton emerges at higher currents. The second order correlation function of the diode demonstrated anti-bunching under a continuous drive current with Hanbury-Brown and Twiss arrangement (Hanbury-Brown \& Twiss, 1956). But the efficiency of collecting the emitted photons is low since the emission from single point diverges in all directions. The reported collection efficiency is about 0.014, and the emitting photons are not at the communication wavelengths. Furthermore, the device emitting single photons with quantum dot is technically complex so that the faint laser pulses are considered as practical single photon sources (Zbinden, 2002).

Optical patch antenna has been proposed for directional emission of single photons and experimentally demonstrated (Esteban et al., 2010; Curto et al., 2010). In their experiment, a single quantum dot emitter is coupled to a nanofabricated Yagi-Uda antenna that resulting quantum-dot luminescence is strongly polarized and highly directed into the high-index glass substrate. Questions are how is the photons reformed or reconstructed so that the divergence changed?

In practical application, the quantum state of a photon is formed by encoding phase information on part of the photon and then recombining the partite so that can be detected at a specified detector (Muller et al. 1997 \& Hughes et al., 2000). That means a dividable photon.

\subsection{Questionable properties of the single photon}

The single photon emitting from single quantum dot can be predicted by the spectrum of the luminescence where only one spectrum line from single exciton exists. The single exciton contains only one electron and one hole so that their recombination can only emit one photon. The results of Hanbury-Brown and Twiss measurement indeed demonstrated no two photons emitted at the same time. However, it can be considered as due to Pauli principle of Fermion, can not be taken as a demonstration of photon antibunching.

The collection efficiency of the single photons needs to be explained. Even the collection efficiency has been increased to about $80 \%$, what is the mean of loss in the photon collection? Is part of the photon lost or the collection is only complete photons but with very low probability. How to increase the efficiency of collecting photons? 
There once more appear the conceptual and philosophical problems of quantum mechanics. There is not clear that if the photons are robust enough so that the collection operation obtains single complete photons by probability, or each of the single photons has lost part of their energy and the detected single photon is somehow reconstructed that is follow the measurement theory developed by Niels Bohr and his colleagues in Copenhagen, saying that it is impossible to separate the quantum mechanical system from the measuring apparatus.

Same question about transmission loss appears: is there exist some single photons they are robust enough until being detected, that the transmission loss of the photons should be quantized based on single photon or all the single photon loses part of their energy gradually until too weak to be detected. The photon has been encoded to an eigen-state so that it should be definitely detected in according to the protocol. The practical results are that the loss increased with transmission distance, and the error bit rate increased also with the transmission distance. For example, in a report, bit error rate of $2.3 \%$ with communication distance of $15 \mathrm{~km}$ rises to $4.1 \%$ with distance of $65 \mathrm{~km}$ (Namekata et al., 2007). This phenomenon can not be simply explained only due to detectors detected more empty pulses after more photon lost in longer transmission distance. This is due to an effect well known as dephase that the transmission photon interaction with environment or quantum permutation. The photon lost part of its energy and combined with equal part of energy with phase unknown from vacuum fluctuation. In fact, there has not definitely been demonstrated that the absorbed photon at the detector is exactly the original one.

Many experiments show that photons are sensitive to environment and the absorption at the detector is a complex process. Many experiments show also that photon emission and absorption usually contain multi-photon interaction, especially in nonlinear process. For example, photons with higher energy down converted to Twin photons in form of entangled states (Neves et al., 2005). Multi-photon absorption has been successfully used for imaging with high resolution and micro-fabrication (Yi et al., 2004). Photons being scattered to emitting a photon in different wavelength such as Raman scattering or Stockes scattering are well-known optical phenomena and extensively been used. For example, single-shot measurement of revival structures of molecules by sequential stimulated Raman transition (Zumuth et al., 2005). Photon emission and absorption are usually in company with the emission or absorption of phonons, for example, photo-acoustic topography has been successfully used for imaging of nanoparticle-containing object (Zh. Yuan, 2005). Multiphoton absorption has been taken as nonlinear phenomenon. The theoretical calculation of three photon absorption is quite agreed with the experiments (Cronstrond \& Jansik, 2004).

Photons are too fragile to be trapped to be study. Nevertheless, the structure of the photon has been considered by Gong Zutong in 1980. a English version of his paper (Gong, 1999) was published for his centennial, but his ideal was from even earlier study in 1933 and based on the elementary quantum theory (Chao, 1933). A photon was thought to consist of a positive and a negative photinos so that can explain many properties of the photon.

The nature of the photon has attracted much attention in recent years. A special issue of Optics \& Photonics was published named "The nature of Light: What is a photon?" in which six feature papers are included (Roychoudhuri \& Roy, 2003).

In the field of quantum information including quantum communication and quantum computation, photons are ideal information carriers that they can be easily coded into different quantum state and transmit a long distance. The most serous problems of the quantum information transmission and processing are due to the dephase and/or 
depolarization that result in quantum bit error rate of more than a few percent in comparison with classical communication where the bit error rate has decreased to less than 10-9. The quantum bit error rate has its special physical origin and need to be studied in more detail. The preparation, transmission and detection of quantum states of the photons in the quantum information system with high fidelity are essential (Combes \&Torner, 2005; Torres \& Torner, 2005; Eiseman et al. 2004).

A concept of reconstruction of photons is introduced in this chapter to discuss quantum state of photon and its detection, to analyze the origin of the bite error, including some technical detail in the single photon detectors.

\section{Quantization of electromagnetic field}

There are mainly two kinds of particles in according to their statistical properties, the Fermion and boson. Photons possess only electromagnetic energy, and mediate electromagnetic interaction. Therefore, photons belong to the type of bosons and are the most ubiguitous bosons. Photons possess discrete energy which can also be deduced from quantization of electromagnetic fields (Yariv, 1988). The total energy of the classical electromagnetic fields, the Hamiltonian

$$
H=\frac{1}{2} \int_{V}(\mu \mathbf{H} \cdot \mathbf{H}+\varepsilon \mathbf{E} \cdot \mathbf{E}) d V
$$

where, $\mu$ is the magnetic permeability, $\varepsilon$ is the dielectric constant, $\mathbf{H}$ and $\mathbf{E}$ are the magnetic vector and electric vector respectively. The integral performs over volume $V$ in consideration. The normal mode expansions of $\mathbf{H}$ and $\mathbf{E}$ are

$$
\begin{gathered}
\mathbf{E}(\mathbf{r}, t)=-\sum_{a} \frac{1}{\sqrt{\varepsilon}} p_{a}(t) \mathbf{E}_{a}(\mathbf{r}) \\
\mathbf{H}(\mathbf{r}, t)=\sum_{a} \frac{1}{\sqrt{\mu}} \omega_{a} q_{a}(t) \mathbf{H}_{a}(\mathbf{r})
\end{gathered}
$$

respectively, where $\omega_{a}$ is the radian oscillation frequency of the ath mode. The normal modes are normalized to meet the orthogonal condition:

$$
\begin{gathered}
\int_{V} \mathbf{H}_{a} \cdot \mathbf{H}_{b} d V=\delta_{a, b} \\
\int_{V} \mathbf{E}_{a} \cdot \mathbf{E}_{b} d V=\delta_{a, b}
\end{gathered}
$$

The magnetic vector and electric vector $\mathbf{H}$ and $\mathbf{E}$ in Eq.(3) are substituted with their normal mode expansions Eq.(4) and Eq.(5) leading to

$$
H=\sum_{a} \frac{1}{2}\left(p_{a}^{2}+\omega_{a}^{2} q_{a}^{2}\right)
$$


Therefore, the Hamiltonian of the electromagnetic fields equals to a sum of harmonic oscillator Hamiltonians. The variable $p_{a}$ canonically considered as momentum, and $q_{a}$ is the canonical coordinate. They are conjugate variables of a quantum mechanical harmonic oscillator, connected by the commutator relations:

$$
\begin{gathered}
{\left[p_{a}, p_{b}\right]=\left[q_{a}, q_{b}\right]=0} \\
{\left[q_{a}, p_{b}\right]=i \hbar \delta_{a, b}}
\end{gathered}
$$

Similar to the quantum mechanical harmonic oscillator, the creation operator $a_{l}^{+}$and the annihilation operator $a_{l}$ for the electromagnetic fields can be defined:

$$
\begin{aligned}
& a_{l}^{+}(t)=\left(\frac{1}{2 \hbar \omega_{l}}\right)^{1 / 2}\left[\omega_{l} q_{l}(t)-i p_{l}(t)\right] \\
& a_{l}(t)=\left(\frac{1}{2 \hbar \omega_{l}}\right)^{1 / 2}\left[\omega_{l} q_{l}(t)+i p_{l}(t)\right]
\end{aligned}
$$

Solving these equations for $p_{l}$ and $q_{l}$ which are inserted into Eq.(8), the result is

$$
H=\sum_{l} \hbar \omega_{l}\left(a_{l}^{+} a_{l}+\frac{1}{2}\right)
$$

There has an important Hermitian operator $\hat{n}_{\mathbf{k} s}=a_{l}^{+} a_{l}$, its eigen value is an positive integer (Mandel \& Wolf, 1995).

$$
\hat{n}_{\mathbf{k} s}\left|n_{\mathbf{k} s}\right\rangle=n_{\mathbf{k} s}\left|n_{\mathbf{k} s}\right\rangle
$$

where, the subscript $\mathbf{k}$ represents the wave vector, and $s$ represents the polarization. $n_{\mathbf{k} s}=0,1,2, \cdots, \infty$. Therefore, the state vector $\left|n_{\mathbf{k} s}\right\rangle$ is number state, or Fock state. The ground state $|0\rangle$ is called as vacuum state. The energy in the vacuum state is not really zero,

$$
\langle 0|H| 0\rangle=\frac{1}{2} \sum_{l} \hbar \omega_{l}
$$

It is an average value over all possible frequencies. It actually represents the vacuum fluctuation, important in the quantum information theory. The annihilation and the creation operators acting on the Fock states result in one photon change in the states that

$$
\begin{aligned}
& a_{l}\left|n_{k}\right\rangle=n_{l}^{1 / 2}\left|n_{k}-1\right\rangle \\
& a_{l}^{+}\left|n_{l}-1\right\rangle=n_{l}^{1 / 2}\left|n_{l}\right\rangle
\end{aligned}
$$

The probability distribution of photons in a coherent state is the Poisson distribution as shown in Eq.(2). 


\section{Optical modes}

The detector detects energy so that especially suitable for Fock state study. In an idealized case absorbing one photon leads to one transition of the electron that releases one pair of electron and hole charge carriers. The quantum efficiency is said to be $100 \%$. The actual emission and absorption are more complex as described in the section 2 . While in theoretical study, it is usual to consider single mode light field interaction with two level atomic systems. However, compared with electron levels, optical modes have much more different features.

\subsection{Interaction of electron with optical fields}

In study on the interaction between the radiation fields and the atom system, the Hamiltonian describing interaction between the electromagnetic fields and an electron (neglecting the electron spin) is

$$
H=H_{\text {Elec }}+H_{\text {Inter }}+H_{\text {Field }}
$$

Where, $H_{\text {Elec }}$ refers to electron motion without the external electromagnetic field. $H_{\text {Inter }}$ is interaction of the electron with the light field. $H_{\text {Field }}$ is the Hamiltonian of the light fields. The interaction of the electron with the light fields may be written in two parts as (Walls \& Milburn, 1994)

$$
\begin{aligned}
& \hat{H}_{\text {Elec }, 1}=\int \Psi^{+}(x)\left(-\frac{e}{m} \mathbf{A} \cdot \mathbf{p}\right) \Psi(X) d^{3} x \\
& \hat{H}_{\text {Elec }, 2}=\int \Psi^{+}(x)\left(-\frac{e^{2}}{m} A^{2}\right) \Psi(x) d^{3} x
\end{aligned}
$$

where, $\mathbf{A}$ is the vector potential of the electromagnetic field, $\mathbf{p}$ is the momentum of the electron, $e$ and $m$ are the charge and mass of the electron respectively. The electromagnetic field operator expressed as a superposition of the unperturbed wave functions that

$$
\Psi(x)=\sum_{j} a_{j} \varphi_{j}(x)
$$

Though the Fock state and number operator can be used to study photon emission and absorption, explain quantum collapses and revivals for interaction of a two-level atom with a single mode field. It is not good enough for practical situation or from a rigorous theoretical treatment in consideration of the reasons:

1. The size of a quantum dot is very small so that in consideration of the uncertainty principle of quantum mechanics the light emitting is omnidirectional, it has a large divergence angle.

2. The absorption happens in a very short time so that the emitted photon has a large bandwidth in according to the uncertainty principle of the quantum mechanics.

3. Even for the polarization, it is always considered as combination of two parts or two orthogonal modes so that a horizontal linearly polarization light is a sum of two linear polarizations orthogonal each other making a $45^{\circ}$ angle to the horizontal or a sum of right and left circularly polarized light. 
4. Optical modes should be considered as a position similar to levels for electron in an atomic system. The different is that a level allows only one electron to occupy, while an optical mode can be occupied by many photons, and the number in one optical mode has no limitation yet. One photon can also be shared by several optical modes as doing in quantum information where information is coded to part of the photon in different mode. One photon shared by four optical modes shown as multipartite entanglement was experimentally demonstrated. Therefore, a part of one photon exists in one optical mode should not considered as "probability of finding one photon", that is indeed a component of the photon (Papp et al., 2009).

Therefore, we should consider that one photon consists of multi-components existing in different modes, or shared by multi-modes. It should be noticed that the number operator is obtained from the integral of a set of normal modes which describe field distribution. One photon should be expresses as a sum of a complete set orthogonal normalized eigen functions. We therefore prefer to start from the studying the fundamental modes of the electromagnetic fields.

\subsection{Quantization conditions of planar waveguides}

The fundamental modes are characterized by the quantization of the vectors appeared in the wavefunction including polarization and the wave vector. To find the eigen value, Einstein proposed a generalized quantization rule (Stone, 2005, as cited in Einstein, 1997)

$$
\oint_{C_{i}} \mathbf{p} \cdot d \mathbf{q}=n_{i} h, \quad i=1,2,3, \cdots
$$

where, $\mathbf{p}$ is momentum, $\mathbf{q}$ is coordinate, $\mathrm{h}$ is the Planck's constant, $\mathrm{C}_{\mathrm{i}}$ is a closed independent loop. This formula is correct in deal with angular momentum. Though the Einstein quantization condition may need a small modification in dealing with practical system including no central forces, this formula is fundamental correct if the boundary condition is properly considered. For example, in dealing with a symmetrical planar dielectric waveguide, the integral form of the quantization condition changes to summation form that phase changes at the boundary have to be added to the summation (Saleh \& Teich, 1991). The quantization condition is

$$
2 k_{y} d-2 \varphi_{r}=2 \pi m, \quad m=1,2,3, \cdots
$$

where $k_{y}$ is the $\mathrm{y}$-component of the wave vector, $\mathrm{d}$ is the geometrical thickness of the waveguide in the $y$-direction, the wave is guided to the $z$-direction. The Einstein's formula holds still if we use the effective optical length $d_{\text {eff }}=d-\varphi_{r}$. The phase change of reflection at boundary can be obtained from Fresnel Equations

$$
\begin{gathered}
r_{T E}=\frac{n_{1} \cos \theta_{1}-n_{2} \cos \theta_{2}}{n_{1} \cos \theta_{1}+n_{2} \cos \theta_{2}} \\
r_{T M}=\frac{n_{2} \cos \theta_{1}-n_{1} \cos \theta_{2}}{n_{2} \cos \theta_{1}+n_{1} \cos \theta_{2}}
\end{gathered}
$$

These formulae show different polarization has different reflection coefficient. $r_{T E}$ is the reflection of the TE waves. The direction of the electric component of the TE modes keeps 
unchanged at the boundary while the magnetic components and wave vector change their directions in the incident plan so that called transverse electric light waves. TM is called the transverse magnetic light waves or TM polarized light waves for the same reason. It is convenient to define $\xi_{j}=n_{j} k_{0} \cos \theta_{j}, \quad j=1,2$, to rewrite Eg.(22) as

$$
\begin{aligned}
& r_{T E}=\frac{\xi_{1}-\xi_{2}}{\xi_{1}+\xi_{2}} \\
& =\frac{\left(\xi_{1}-\xi_{2}\right)^{2}}{\xi_{1}^{2}-\xi_{2}^{2}}=\left[\frac{\xi_{1}+i\left(i \xi_{2}\right)}{\sqrt{\xi_{1}^{2}+\left(i \xi_{2}\right)^{2}}}\right]^{2}
\end{aligned}
$$

The same calculation can be performed for TM waves that leads the reflection from medium $a$ at $a / b$ boundary can be written as

$$
r_{a b} \equiv \exp \left\{-2 i \varphi_{a b}\right\}
$$

where,

$$
\varphi_{a b}=\arctan \left(\frac{\eta_{a} \xi_{b}}{i \eta_{b} \xi_{a}}\right)
$$

where the coefficients of the polarization

$$
\eta_{k}=\left\{\begin{array}{cc}
1, & T E \\
n_{k}^{2}, & T M
\end{array}\right.
$$

Therefore, not only the wave vector quantized, the polarization modes are also nondegenerated.

\subsection{The fundamental modes in free space}

Photons mediate electromagnetic interactions which also lead to quantization of the physical observables similar to Coulombic interaction in an atomic system. The electromagnetic interactions include coherent interference and coherent combination. The electromagnetic interaction happens during their superposition.

It is reasonable to start from consideration of polarized monochromatic optical waves. We consider TM mode that the direction of the magnetic vector of the optical wave will not change while the electric vector and the wave vector can change their directions in a plan containing them. The resonant condition considered in the phase space is

$$
r \frac{d k}{d \theta} d \theta=-k d r
$$

The photons survived in resonance. This is why a lens can focus an optical beam: the relative phase delays are compensated by direction change of the wavevector so that the curvature of the wavefront changes as shown in Fig.1. There exists angular momentum due to strong electromagnetic interaction. 


$$
\mathbf{J}=\mathbf{r} \times \frac{\partial \mathbf{k} \hbar}{\partial \theta}
$$

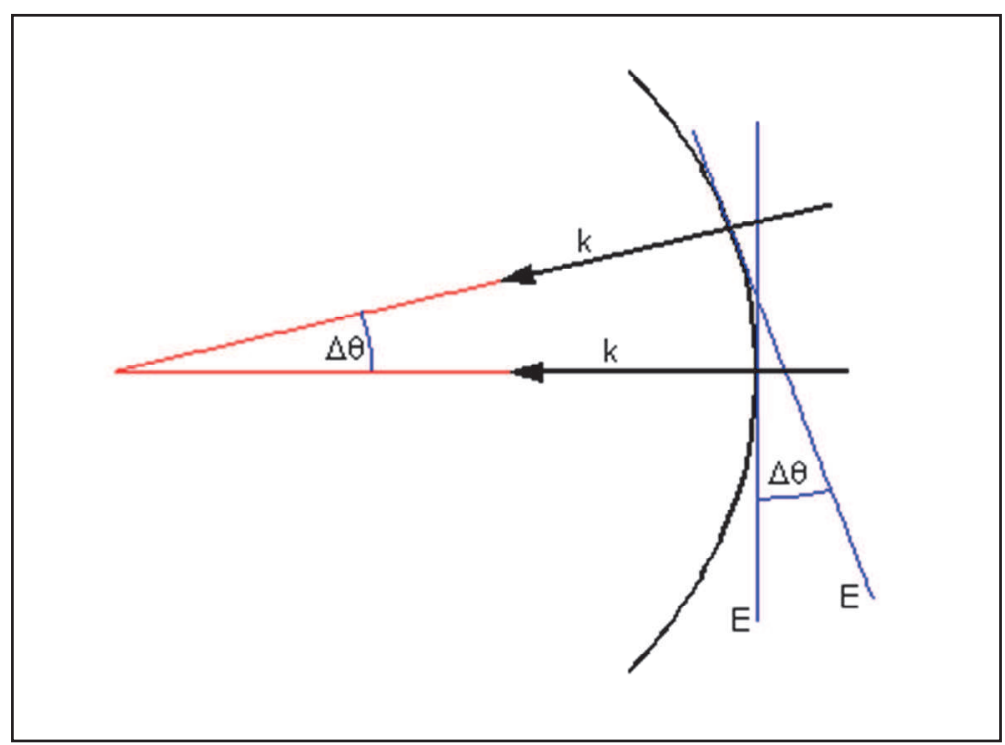

Fig. 1. Angular momentum requires the wavevectors changing their direction in a plane perpendicular to the angular momentum to meet the resonance condition. The relative phase delay along a distance is compensated by electric field vector rotation of an angle equal to that of the $\mathrm{k}$ vector rotation of $\Delta \theta$.

To deal with angular momentum, the Einstein's generalized quantization rule is correct, used here leading to

$$
\oint \mathbf{p} \cdot d \mathbf{q}=n_{i} h, \quad n_{i}=0, \pm 1
$$

These results indicate three fundamental modes in free space. $n_{1}=0$, corresponding to plane waves. The modes with nonzero angular momentum have curved phase front that the phase delayed monotonically clockwise or anti-clockwise indicating the direction of the angular momentum. This is a result that the coherent superposition of the electromagnetic fields appeared as self-confined field, a condition called self-consistency.

The fundamental modes with non-zero-angular momentum have attracted much attention in recent years. The integral closed with a phase difference of $2 \pi$ for a complete mode with angular momentum equal to +1 or -1 . A complete mode includes all directions of the waves in the plan needed for the loop integral. This mode contains always two oppositely propagating waves. A relative phase delay of $\Pi$ exists between the oppositely propagating waves so that the wave function with non-zero angular momentum should be

$$
\psi_{ \pm 1}=\frac{1}{\sqrt{r}}\left(e^{-i k r}+e^{i(k r+n \pi)}\right), \quad n= \pm 1
$$


where the time-dependent factor $e^{i \omega t}$ being omitted. The intensities of the mode have cylindrical symmetrical distribution as shown in Fig.2

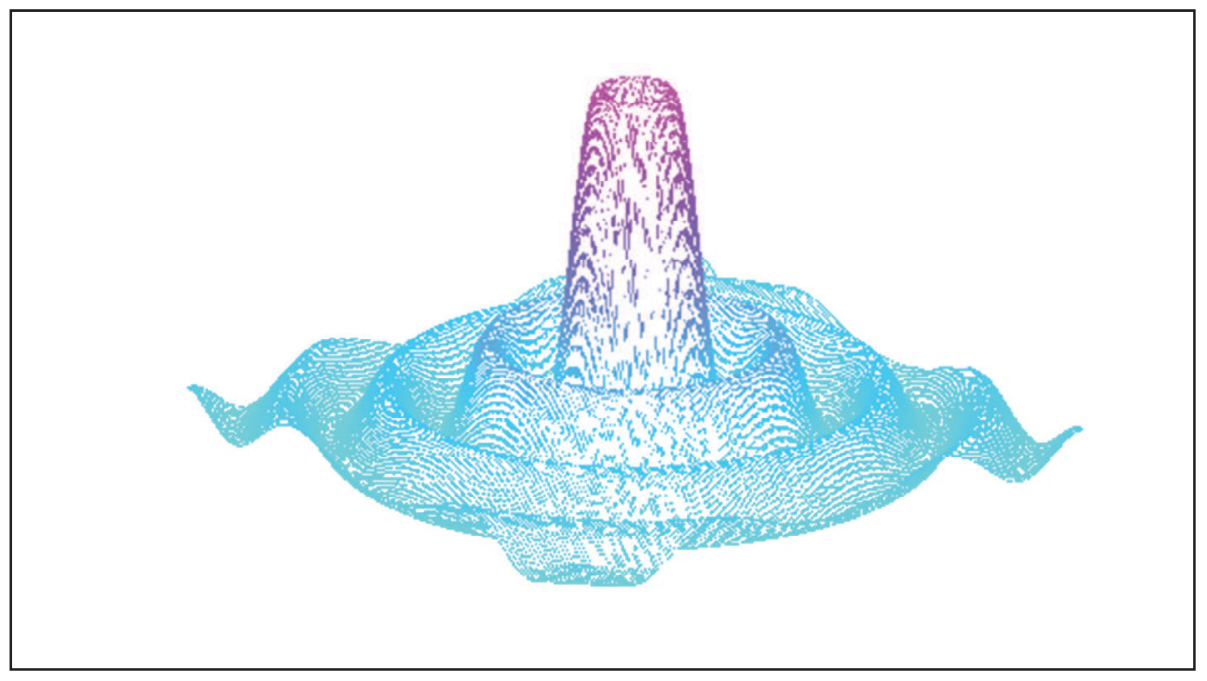

Fig. 2. A complete fundamental mode with angular momentum equal to \pm 1 has cylindrically distribution in space. The intensity vanished at center.

The further quantization is the generation of vortex lattice (Ghai et al. 2009; Wei et al. 2009).

\subsection{Mode structure of an optical beam}

One of the solution paraxial Helmholtz equation is Gaussian beam. In comparison with Hermite-Gaussian beams or Laquerre-Gaussian beams, the Gaussian beam is taken as the fundamental mode or single mode. The intensity distribution of a Gaussian beam is a single spot that is similar to the intensity distribution of the lowest order mode $(0,0)$ of the Hermite-Gaussian beam. A Gaussian beam appears under paraxial waves assumptions. The phase of a Gaussian beam may be expressed (Saleh \& Teich, 1991) as

$$
\varphi(\rho, z)=k z-\zeta(z)+\frac{k \rho^{2}}{2 R(z)}
$$

where, the beam propagates to z-direction, $\rho^{2}=x^{2}+y^{2}$. On the beam center, $\rho=0$, the Eq.(32) represents a plan wave. $R(z)$ is the wavefront radius of the curvature. However, the radius of the curvature changes with distance $z$ that the radius is infinite at $z=0$ where is called beam waist, having a planar wavefront. The radius decreases with $\mathrm{z}$ to a minimum and then increases. The wavefront far from beam waist is approximately the same as that of a spherical wave. The Gaussian beam has a intensity distribution similar to that of the lowest order mode $(0,0)$ of the Hermite-Gaussian beam, and therefore be called as single mode beam. However, the variation of the radius of the wavefront curvature could not be explained by mode theory. A single mode requires a constant eigen value for the 
momentum. A fundamental mode based on self-consistency guarantees shape invariance. The variation of the wavefront curvature could only be explained by interaction between different modes.

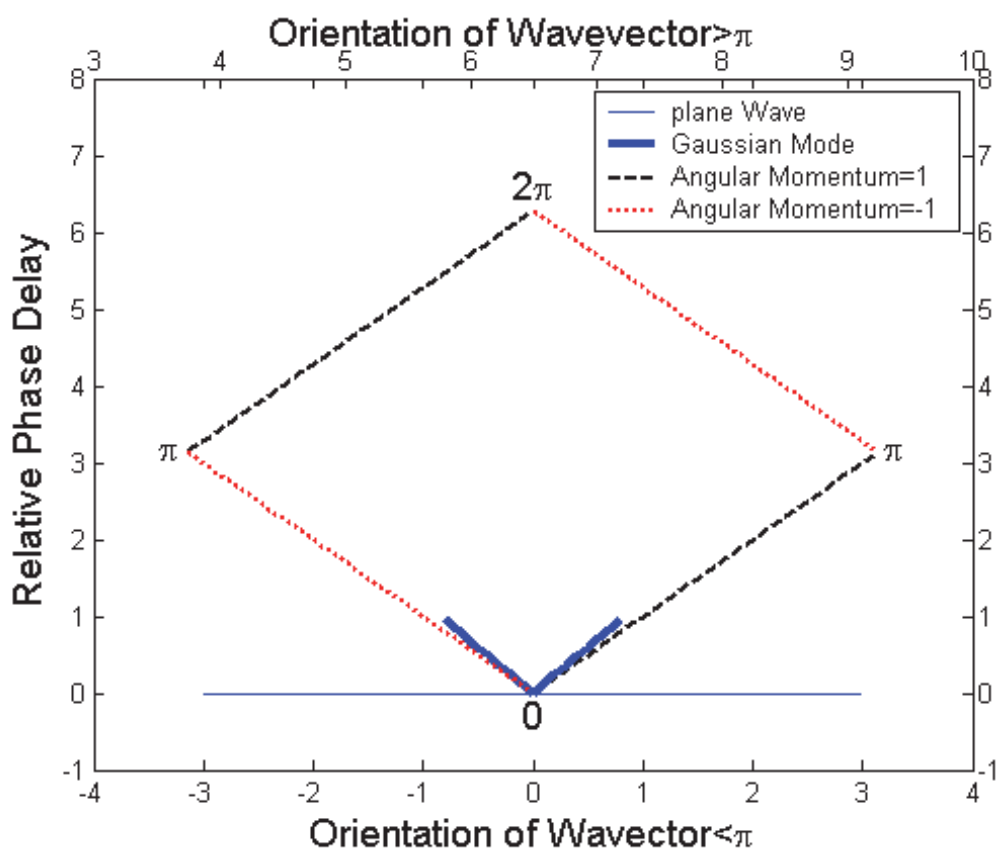

Fig. 3. The phase distribution of a Gaussian beam at a position far from its beam waist where it has a spherical phase front. Compared with the fundamental spatial phase which monotonically delayed clockwise or anti-clockwise indicating the direction of the angular momentum, the Gaussian beam (sick line) starts at its center increased its spatial phase all the same in both direction and limited to a small area surrounding the beam axis due to the paraxial assumption. The thin line represents plan wave.

The phase distribution of a Gaussian beam at a position far from the beamwaist has approximately spherical wavefront. The spatial phase of the Gaussian beam, fundamental modes with angular momentum equal to $0, \pm 1$ are shown in Fig.3. The fundamental modes with non-zero angular momentum increase or decrease their phase monotonically to close with a phase difference of $2 \pi$. A Gaussian beam increases its spatial phase all the same at both sides each belongs to different angular momentum. It is the interaction between the two modes decides the variation of the wavefront of the Gaussian beam. The interaction between the two fundamental modes in a Gaussian beam is depicted in Fig. 4.

A Gaussian beam is usually produced by a cavity formed by spherical mirror resonators with a small aperture as output mirror. The output beam has a wavefront similar to spherical waves. A spherical wave output from the mirror is that phase delayed at the center and then increasing its spatial phase with distance in the radius direction. In the consideration of fundamental modes, if the output is polarized light, it contains nonzero angular momentums belong to both electric vector and the magnetic vector respectively so 
that there are four fundamental modes at the least. Under the paraxial assumption for the optical beam, the light within a neighborhood of size $\lambda$ is locally like a plan wave, while the Gaussian beam actually posses paraboloidal wave at far from the beam waist.

Interaction of theses modes result in the paraboloidal wavefront. We consider one polarization in a plan containing wavevector and the wavefront of two fundamental modes with angular momentum equal to \pm 1 as depicted in Fig.4. The two modes keep in phase at the center, while the phase difference increases with the distance to the beam center. That is the destructive interference increases with this distance. This is the reason a Gaussian beam can confine spatially and transport light energy through free space to a long distance. However, two or more Gaussian beams can not coherently combine all together, instead, they produce interference pattern.

Optical beams that belong to same fundamental mode can topologically combine to form optical vortices that are promise to provide extreme high power density needed for fundamental study at extreme conditions. It is also a possible scheme for efficient laser fusion.

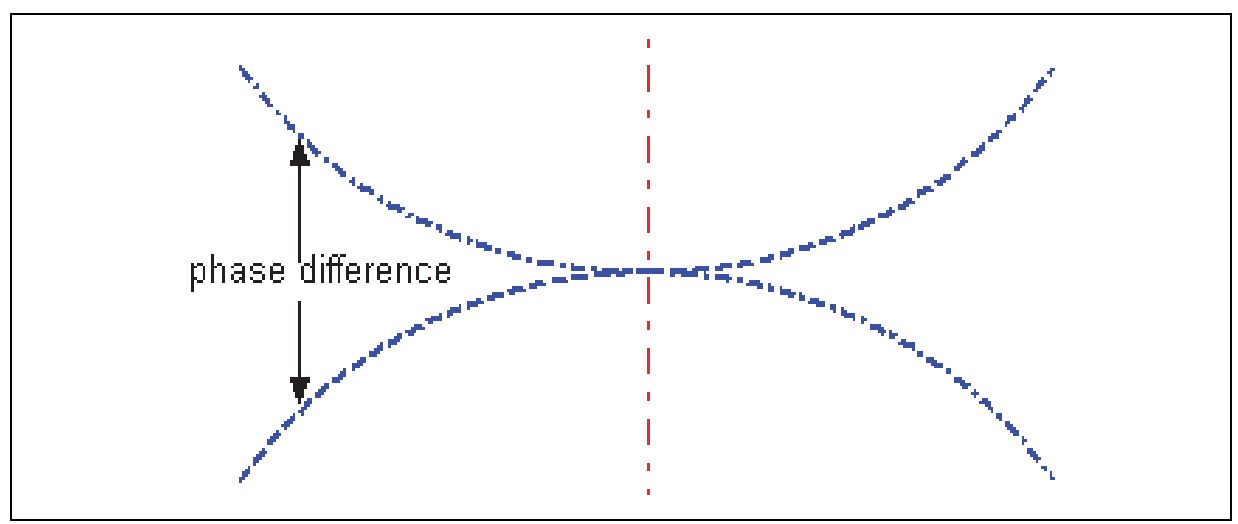

Fig. 4. A Gaussian beam has nearly a spherical wavefront far from its waist where two modes combine at center keeping in phase and then the phase increases in both directions clockwise and anti-clockwise, the phase difference between the two fundamental modes increased also. Dashed line and dot-dashed line represent different modes respectively.

Two or more light beams each belongs to different fundamental mode may be coherently combined via unitary transformation. The unitary transformations are actually mode transform. However, for the Gaussian beams, their complex mode structures prevent this kind of transformation. The number of the modes increases with the number of the Gaussian beams, only components belong to single fundamental mode can be combined while the others have both constructive and destructive interference that lead to complex pattern.

An analysis of a focusing Gaussian beam indicates that it has wavevector on the beam axis carrying plane waves surrounded with declined wavevectors. Looking at a plane perpendicular to the beam axis, there are components of these vector pointing to the beam center from all possible direction. With a proper topological charge, these vectors are topologically combined to form optical vortex. However, the optical vortex is appeared in 
first order beam. In an experiment, the formed optical vortex has to be separated from $0^{\text {th }}$ order beam for measurements. The $0^{\text {th }}$ order beam still carries the component of the plane wave and it is much stronger (Yang, 2009).

\section{Bunching and antibunching of photons}

Photons have a trend to have collective behavior such as in the stimulated amplification. Even in a faint laser pulse, the multi-photon events could not disappear. However, in the quantum statistics, there exists indeed antibunching and sub-Poissonian statistics. Antibunching predicts that photons come one after the other that are single photons. Single photons are extreme important for quantum key distribution that guarantees the security of the communication. Photon anti-bunching effect is also important for understanding photons since the photon anti-bunching is regarded as a fully quantum mechanical phenomenon without a classical analogue (Pathak \& Mandal, 2003).

The photon anti-bunching is the quantum statistical property. It is usual to consider the second order correlation function of a single-mode field for the photon statistics. The second order correlation $g(2)(0)$ factor with zero-time delay is defined as

$$
g^{(2)}(0)=\frac{\left\langle a^{+} a^{+} a a\right\rangle}{\left\langle a^{+} a\right\rangle^{2}}=1+\frac{V(n)-\bar{n}}{\bar{n}^{2}}
$$

where, $V(n)=\left\langle\left(a^{+} a\right)^{2}\right\rangle-\left\langle a^{+} a\right\rangle^{2}$. For a coherent state, $g^{(2)}(0)=1$ and $V(n)=\bar{n}$, the number state has a Poissonian distribution. A classical analysis predicts always $g^{(2)}(0) \geq 1$, there is a tendency of photon bunching. For $g^{(2)}(0)<1$, it is the anti-bunching which is a feature peculiar to the quantum mechanical nature of the electromagnetic field (Walls \& Milburn, 1994).

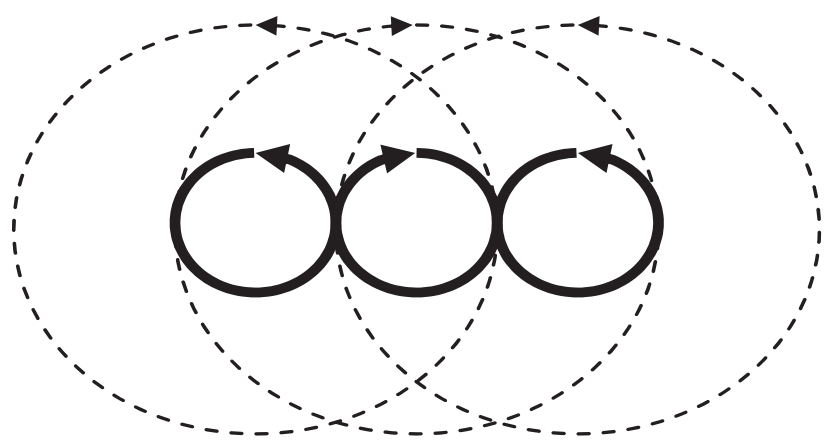

Fig. 5. Optical vortices with angular momentum $= \pm 1$ refuse to share same position in space that can be considered as spatial antibunching.

The explanation of the photon anti-bunching is challenge. The macroscopic electromagnetic interaction show coherent combination and coherent interference phenomena. Therefore the anti-bunching were explained as the particle natures of the photons. Many models have been proposed to possess anti-bunching effect. Among several experiments containing 
interaction of light field with electron or atomic systems that demonstrated anti-bunching light, the most promising is the electrically driven single-photon source (Zhiliang Yuan et al., 2002). In their experiment, the HBT measurement demonstrated indeed sub-Poissonian photon statistics and anti-bunching. Most of the experiments reported to date demonstrate antibunching using radiation field interaction with atomic or ion system which limit the number of the photon emitting. Is there any reason that from the property of the photons by themselves that should result in antibunching? Why the photons can distinguish each other? Destructive two-photon interference demonstrated photon antibunching with calculated $g^{(2)}(0)<1$. Actually, they obtained both the bunching and antibunching by controlling the phase difference between two input beams so that the production process is difficult to understanding by a naïve photon picture (Kaoshi \& Matsuoka, 1996).

To answer these questions, one should also consider the physical observables that are quantized wavevectors or polarization vectors. Electromagnetic fields never refuse to interfere with each other. There are two cases that should be considered. One is the coherently combination, the other is the interference coherently. If we consider the monochromatic wave as single mode, the two linearly polarization orthogonal each other to form a new state only when their wave vectors lay on line. For the linearly polarized light with wave vector in different direction in same plan, they can coherently interfere to produce fringes when across each other, while keeps their independent so that keep their polarization and wave vector unchanged after crossover. However, if these waves topologically charged they can combine to share a same angular momentum. The optical vortices formed under strong electromagnetic interaction where the wavevectors topologically combined. The optical vortex with angular momentum equal to 1 refuses to take same position with the optical vortex with angular momentum equal to -1 in the space so that vortex lattice formed as shown in Fig.5. This phenomenon can be considered as spatial anti-bunching. However, there should be collective behavior of large amount of photons since the optical vortices are the results of strong electromagnetic interaction provided by photons themselves. Here, the optical vortices with different angular momentum can distinct each other. The optical vortices are now mostly considered due to orbital angular momentum.

\section{Directional emission of photons}

The divergence of photon emitting from a cavity can be easily estimated from uncertainty principle of the quantum mechanics.

$$
\Delta x \Delta p_{x} \geq \frac{\hbar}{2}
$$

Supposing the size of the antenna is $R=\Delta x$, the divergence angle $\theta=\Delta p_{x} / p_{x}$ and $p_{x}=\hbar k=2 \pi \hbar / \lambda$, it is immediately to have

$$
\theta=\frac{\lambda}{4 \pi R}
$$

Therefore, the divergence angle increases with decreasing the size of the antenna. The divergence angle of photon emitting from quantum dots is very large that a photon shared by all possible modes. As a result, the collection efficiency of the photon is very low. 
Put a quantum dot in cavity or waveguide that may limit the photon to emit only to desired modes. But that is difficult in technical realization. Using surface plasmon resonances to form optical patch antennas is promise (Esteban et al., 2010). The unidirectional emission of a quantum dot coupled to a nanoantenna was experimentally demonstrated (Curto et al., 2010). In their experiment, a quantum dot was placed in the near field of one of the fiveelement gold Yagi-Uda antennas for operation wavelengths of $\sim 800 \mathrm{~nm}$. The total length of the antennas is $830 \mathrm{~nm}$. The antennas emission a beam with an divergence angle at half maximum of $12.5^{\circ} \times 37^{\circ}$ pointing into the glass substrate. The simulations indicated that as much as $83.2 \%$ of the QD emitted light were collected.

This coupling demonstrates a mode selection. The surface plasmon can only be excited by TM waves. As a result, quantum dot emission is transformed onto TM mode via surface plasmon resonance. The interaction of the five-element emission decided the direction of the optical beam. The mode selection is from two physical reasons:

1. Photons are survived in resonance. Once the TM mode resonant with surface plasmon, it absorbs all energy of the photon possess, TE mode is depressed.

2. The interaction of the radiation field emitted by the antennas decided the emission direction and divergence. That is decided the mode structure of the photon.

Divergence from an emitter can not be avoidable. The detector requires convergence of the beam. There is still a need to study the wavefronts and the mode structure, if one considers the reciprocity: that is if a next similar five-element Yagi-Uda can sense the emitted beam so that results an absorption in a quantum dot under the assumption adiabatic unitary transformation?

There has no evidence that an adiabatic unitary transformation is technical realistic. Loss and dephase are unavoidable. However, many experiments have demonstrated with single photon detector now available commercially, many quantum information experiments can be successfully performed.

\section{Single photon detector}

Single photon detectors are essential for quantum information applications. Single photon detectors at the communication wavelengths have attracted much attention in recent years. Here we introduce some of the results on developing single photon detector at communication wavelengths in our laboratory.

\subsection{Characterizing an APD for single photon detection}

Single photondetectors used in quantum key distribution ask for very high sensitivity and extreme low noise. The InGaAs avalanche photodiodes (APD) are usually chosen for single photon detection at the infrared communication wavelengths. This APD has a structure of separate absorption, grading, and multiplication (SAGM). The SAGM APD has been studied extensively and successfully used for single photon detection in the infrared communication wavelengths. The structure of this APD is depicted in Fig.6. The absorption layer InGaAs is designed to have a bandgap of $0.73 \mathrm{eV}$ so that the sensitivity can extend to about $1650 \mathrm{~nm}$. A grading layer between the absorption layer and the multiplication layer facilitates the holes induced by absorption of photons to transit into the multiplication layer (Hiskett et al., 2000). 


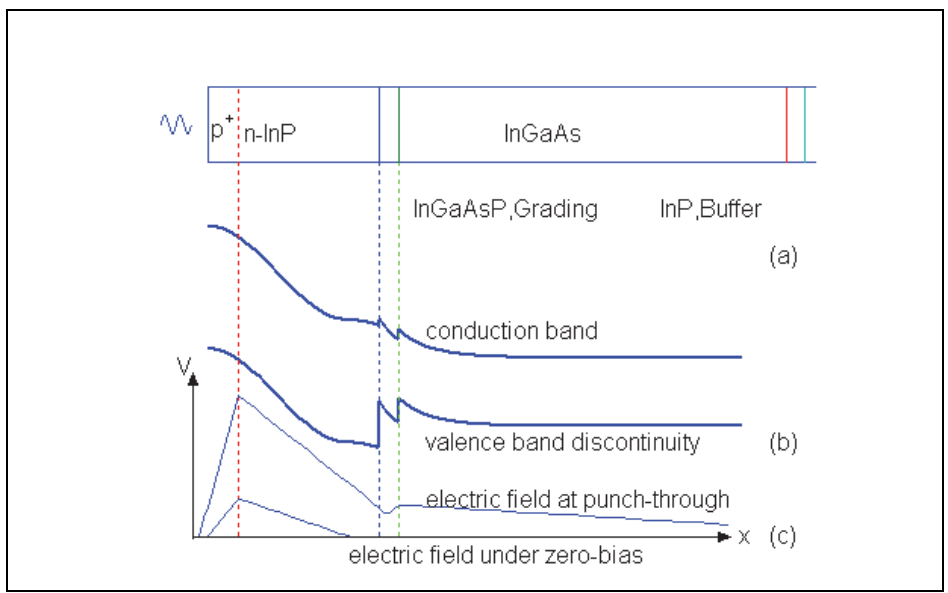

Fig. 6. (a) The structure of the SAGM APD, (b) the band diagram of the APD, (c) The inner build electric field under zero bias and under punch-through.

This APD has to be operated in Geiger mode to exploit the extreme sensitivity. A Geiger mode means the APD is operated at a bias higher than its breakdown voltage that any carrier in the multiplication layer will initial self-sustained avalanche. As a result, the APD should have extreme low dark current. Therefore, there are only a few choices of APDs commercially available for operation in Geiger mode. The APDs have to be operated at low temperature. The APD will be damaged if there is not quenching voltage immediately after the avalanche to stop the self-sustained current. Therefore, the Geiger mode is usually realized in gated mode operation. The gated pulses applied on the APD are synchronized with the arriving of the signal photons.

However, the breakdown voltage itself is not very clearly defined. Theoretically, breakdown voltage is said at that voltage the multiplication factor goes to extreme large, or selfsustaining avalanche appeared. But in experiment, it usually included various guess work. For example, the breakdown voltage is defined as the bias voltage at which dark current is $100 \mu \mathrm{A}$ (Maruyama et al., 2002). Or, the voltage when the first pulse with peak value of 100 $\mathrm{mV}(0.5 \mathrm{mV}$ at the APD) was detected (Rarity et al., 2000). The voltage higher than the breakdown voltage is called excess voltage or the relative excess bias. Exploring the use of the excess voltage for higher sensitivity has also been reported. Therefore, the characterization of the APD at voltage higher than the breakdown is also needed. However, the characterization of the I-V curves is usually stopped at the guessed breakdown voltage to prevent APD from being damaged. Here we introduce the I-V characterization including the excess voltage with breakdown voltage well defined by actual measured value.

A passive quench circuit with a $200 \mathrm{~K} \Omega$ quench resistor is used to characterize an APD of type C307645E from EG\&G. The APD was cooled to a temperature of $-25^{\circ} \mathrm{C}$ by Peltier effect. A $1.31 \mu \mathrm{m}$ pigtailed DFB diode laser attenuated to $-45 \mathrm{dBm}$ was used as input signals which switches on to measure photon-current-voltage curves, and switches off to measure darkcurrent-voltage curves. The measured results are shown in Fig.7 which is very similar to other corresponding reports.

The punch through voltage and the breakdown voltage are not clearly indicated in the I-V curve shown in Fig.7. We define a parameter called relative current gain 


$$
G_{r}=1+\frac{d I}{d V}
$$

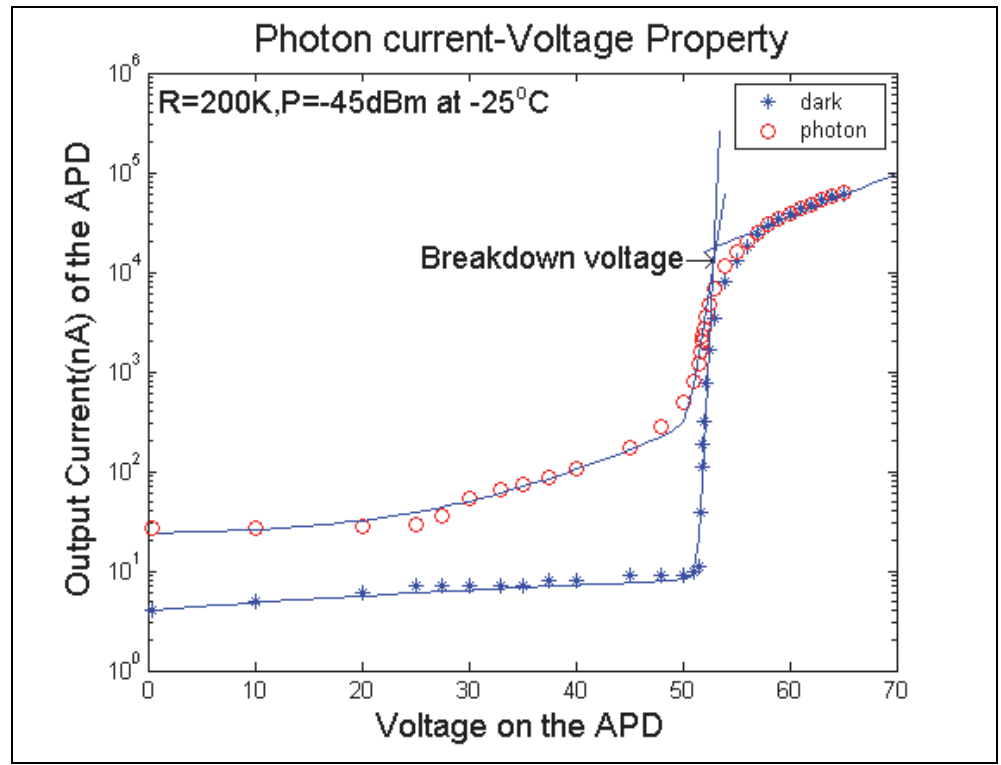

Fig. 7. The breakdown voltage and the bias were decided from these I-V relations experimentally.

The reason is obvious. The multiplication factor has reached it maximum after breakdown, the gain is saturated. The detector performs as a linear device before avalanche and after breakdown. The relative current gains are plotted with the applied voltage both for the photon-current and the dark current as shown in Fig.8 where the data are the same as in Fig.7. The breakdown voltage and the punch through voltage are much more clearly indicated.

The breakdown voltage makes no difference between the photon induced carriers and the dark carriers. But the dark carriers start to avalanche at higher bias. This features a depletion region exists at the vicinity of the heterojunction of the InGaAs/InGaAsP.

\subsection{Operation parameters of APD for better performance}

It is usual in designing a single photon detector, the operating voltage and the temperature should be carefully considered. The operating voltage should higher than the breakdown, that is an excess voltage is needed. With increasing the excess voltage, the sensitivity seems to be increased, but the error bits or dark counts increased also. There is still uncertain that how high the excess voltage is the best. Thermal excitation decreased with cooling the temperature. It seems the lower temperature is the better. But from the consideration of practical application and the phenomenon the dark carriers not start to avalanche immediately after punch-through, it is reasonable to optimize the operation temperature. 


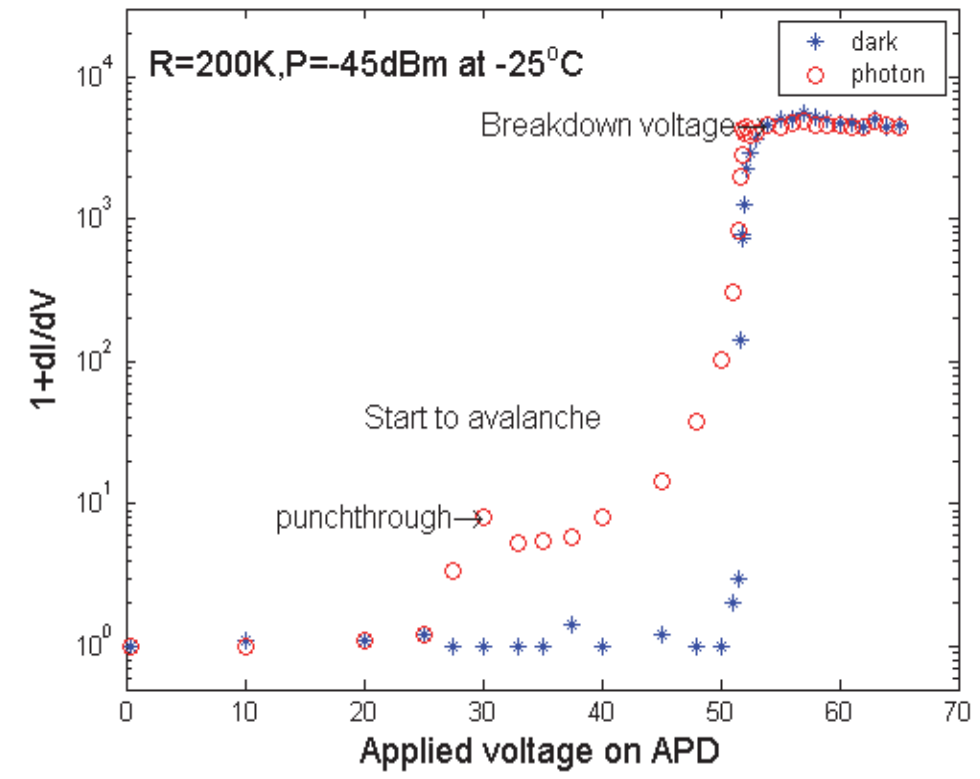

Fig. 8. The relative current gain versus bias characterization curves clearly indicating the punch through voltage, breakdown voltage and the avalanche.

In according to the I-V property shown in the Fig.8, one should consider both the bias and the operation temperature at the same time. Because the breakdown voltage decreases with temperature, the breakdown voltage can be adjusted by cooling the APD so that it is larger than the punch through voltage but not too high to avoid the breakdown initiated by dark carriers. In the Fig.8, the breakdown voltage of about $50 \mathrm{~V}$ is a reasonable choice. The excess voltage needed is very limited.

It is well known that the photon absorption follows an exponential law while the thermogenerated carriers follow Gaussian distribution. A calculation analysis is shown in Fig.9 where we calculated the distribution for incident of average $0.1,0.3,0.5$ photons per pulse and suppose the thermo-generated carriers in the pulse duration are 1, 0.75, and 0.5, corresponding to the curves (a), (b), and (c) respectively. The S/N ratio is defined as the photon-induced carriers divided by thermal carriers that can drift into the multiplication layer. It is clear that the $\mathrm{S} / \mathrm{N}$ is fairly high if the bias voltage do not penetrate into the absorption layer too much.

The excess voltage can be controlled by temperature since the breakdown voltage is a function of the temperature. That is the basis the temperature control could be used to adjust the breakdown voltage. The bias larger than that of the punch-through is necessary and the depth of punch through should be carefully chosen. 


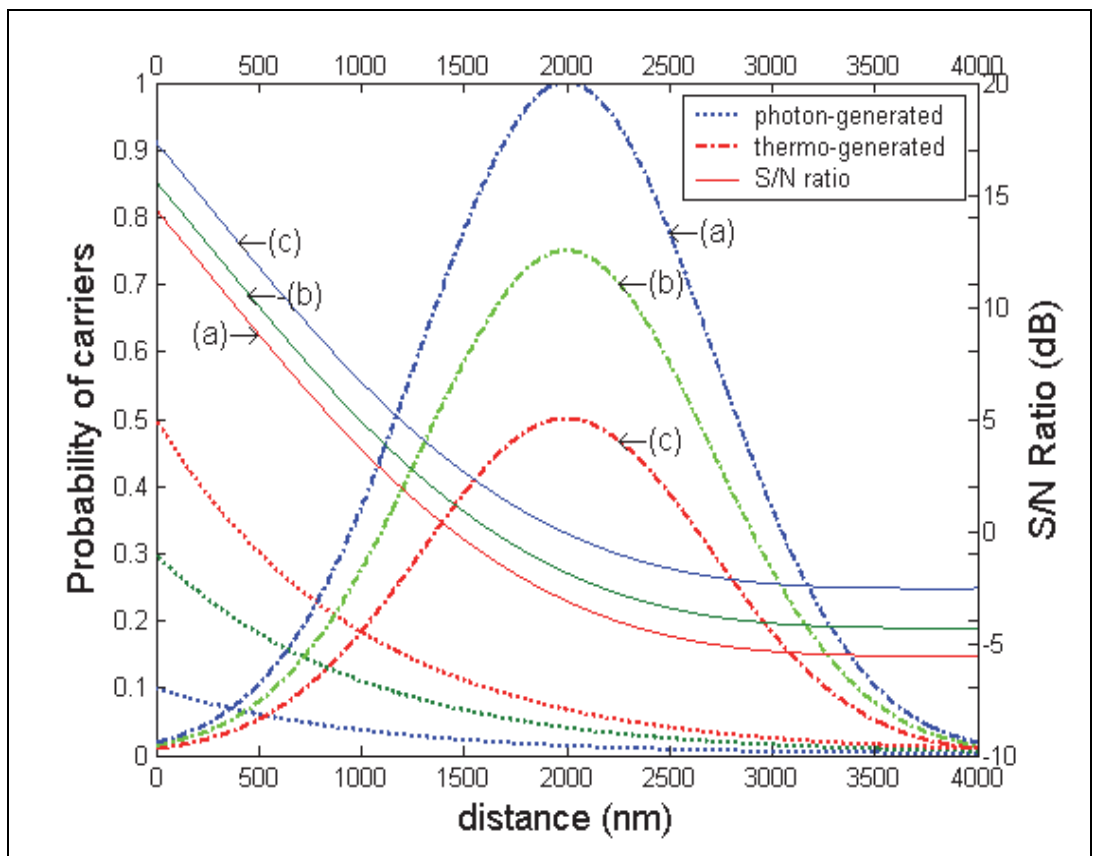

Fig. 9. The spatial distribution of the photon-induced carriers and thermal carriers in the absorption layer calculated on the basis of average number per pulse duration indicating that the depth of the punch-through voltage penetrate into the absorption is very limited for a reasonable $\mathrm{S} / \mathrm{N}$.

\subsection{Integral detection}

The gated electric pulses may produce electric spikes that would result in error counts. Therefore, single photon detector with balanced two APDs has been reported that $\mathrm{S} / \mathrm{N}$ ratio improved by more than one order of magnitude in compared to the conventional usage of APDs (Tomita \& Nakamura, 2002; Kosaka et al., 2003). Although various proposals had added to the balancing structures, they are not only technically complex, the spikes can not be cancelled completely. The integral gated mode single photon detection is much promising for use in quantum key distribution (Wei et al., 2007).

In the method of the integral gated mode detection, an integral capacitor stores the charges of the avalanche current and gives a negative feedback to the APD bias that leads to quench the avalanche at a fixed level. The integral capacitor and a charge amplifier compose as integrator so that the detected signals are static charge on the capacitor. There are no spikes at all and easy for digital processing afterwards.

In the experiments with the integral gated mode single photon detector, the single photon source was attenuated faint pulses with width of 50 ps at $1550 \mathrm{~nm}$ from a gain-switched laser (Sepia PDL808, Picoquant). The APD used in the experiments was from JDS Uniphase (ETX 40 APD BA, ETX00408052-005). The temperature of the APD was stabilized at 224 \pm 0.1 $\mathrm{K}$. The static bias was $43.1 \mathrm{~V}$ which is below the punch through voltage. The gated pulses of $5.13 \mathrm{~ns}$ in FWHM and $4.4 \mathrm{~V}$ peak-to-peak were added to DC bias. The breakdown voltage of 
this APD measured at $224 \mathrm{~K}$ is $46.6 \mathrm{~V}$. Therefore, there is only an excess voltage of $0.9 \mathrm{~V}$. A gate pulse frequency of $100 \mathrm{kHz}$ was chosen in the measurement.

The traces of the APD avalanche recorded by oscilloscope TDS1012 show clearly the transient spike cancellations. A single photon detection efficiency of $29.9 \%$ at dark count probability of $5.57 \times 10^{-6}$ per gate or $10.11 \times 10^{-7}$ has been achieved.

\section{Multipartite entanglement of photons}

The single photon detector records classical information. It records only the energy. In quantum information, there should have records of quantum bits. Therefore, two or more detectors have to be used, and these detectors should be entangled each other. This can be realized with some kind of interferometers. For example, the Mach-Zehnder interferometer as shown in Fig.10, the record at the detector A should be entangled with that at detector B. If detector A records 1, then detector B should record 0 . That is, only record of (10) is correct, while all other records including (01), (00), (11) are error bits.

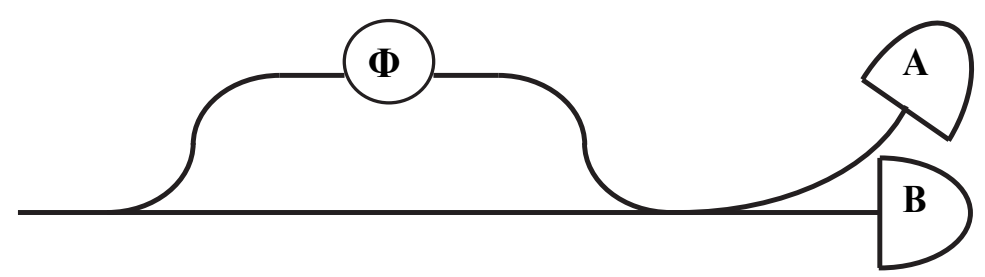

Fig. 10. Mach-Zehnder implementation of quantum key distribution

To obtain a correct record, Bob has to decode by using phase modulation in according to their protocol so that the maximum of the interference is at detector A corresponding to the input quantum signals:

$$
\left(\begin{array}{l}
1 \\
0
\end{array}\right)=\frac{1}{2}\left(\begin{array}{cc}
1 & e^{-i \varphi} \\
-1 & e^{-i \varphi}
\end{array}\right)\left(\begin{array}{c}
1 \\
e^{i \varphi}
\end{array}\right)
$$

One photon can be coherently shared among $\mathrm{N}$ spatially distinct optical modes to form multipartite entanglement, a quantum state being called $\mathrm{W}$ state. A W-state with $\mathrm{N}=4$ can be expressed in the form

$$
|W\rangle=\frac{1}{2}\left[\left(|1000\rangle+e^{i \varphi_{1}}|0100\rangle\right)+e^{i \varphi}\left(|0010\rangle+e^{i \varphi_{2}}|0001\rangle\right)\right]
$$

The multipartite entanglement with $\mathrm{N}=4$ where the partite is formed by beamsplitter has been detected and characterized in more detail (Papp et al., 2009).

A genuine $\mathrm{N}$-partite entanglement is realized only with simultaneous participation of all $\mathrm{N}$ of the constituent systems. There is also a similar case where is $\mathrm{N}$ time-distinct partite entanglement. There are $\mathrm{N}$ time-distinct faint pulses to share one photon. This kind of multipartite entanglements is realized in differential phase shift key distribution system where the simultaneous participation of the constituent systems is due to the nonlocality of the photons. 
However, the utilization ratio of the traditional differential phase shift is low. Their key creation rate under ideal condition can only reach $(1-1 / N)$ if a single photon pulse is split into $\mathrm{N}$ sequential ones. The utilization ratio of photons can reach 1 under ideal condition by discrimination and controlled delay of the first pulse (Wang et al., 2009). This scheme can also result in a genuine $\mathrm{N}$-partite entanglement with $\mathrm{N}$ time-distinct constituents. In the proposed scheme of 6-partite entanglement, the two pulse trains containing three timedistinct pulses in each formed by beam splitter and controlled time delay. The multiplex at Bob side are designed such that with the first single pulse of the first train two-bit delayed the rearranged pulse train can recombine with the next pulse train in three time slots exactly. Their coherent superposition represents an entangled state of a photon in threedimensional Hilbert space with four non-orthogonal states:

$$
\frac{1}{\sqrt{3}}\left(|V\rangle|1\rangle_{A}|0\rangle_{B}|0\rangle_{C} \pm|H\rangle|0\rangle_{A}|1\rangle_{B}|0\rangle_{C} \pm|H\rangle|0\rangle_{A}|0\rangle_{B}|1\rangle_{C}\right)
$$

Where, $|V\rangle$ is vertical polarization state, $|H\rangle$ is the horizontal polarization state. All the constituents of a photon have involved in the key creation so that is a genuine multipartite entanglement state. Not only the key create ratio is increased, the security is also enhanced.

\section{Dephase and decoherence}

Dephase and decoherence are unavoidable even in the case under idealized condition without loss if one consider a rigorous representation of the wavefunction for single photons. In according to quantum mechanics, a state of a particle is represented as a complete set of eigen functions. Therefore, the number state of $\mathrm{N}=1$ should be expressed in a two dimensional Hilber space that

$$
|\psi(1)\rangle=\alpha|0\rangle+\beta|1\rangle
$$

Even in pure vacuum, the fluctuated electromagnetic fields exist that make up the zeropoint energy. Quantum theory predicts that empty space is not truly empty. In an electromagnetic field, virtual photons created and annihilated constantly that make contribution to a small renormalization of the energy of a quantum system, known as the Lamb shift. The experimental observation of the Lamb shift in a solid system has been reported (Frabner et al., 2008). A scheme including vacuum state that can be used to demonstrate the nonlocality of a single photon experimentally has also been proposed (Dunningham \& Vedral, 2007).

In their scheme, classical faint pulse incident on beamsplitter has been expressed as two input ports and two output ports where a state of Eq.(40) and a vacuum state $|0\rangle$ are incident on the two input ports of a 50:50 beamsplitter, and two output state of U1 and U2 as shown in Fig.11. It is obvious that vacuum fluctuation in the output state is increased. In their model, they have chosen the particular values that $\alpha=1 / \sqrt{3}$, and $\beta=\sqrt{2 / 3} e^{i \varphi}$ to simplify the analysis without losing the generality of their arguments. The state after the beamsplitter is

$$
|\psi\rangle=\frac{1}{\sqrt{3}}\left[|0\rangle|0\rangle+e^{i \varphi}(|0\rangle|1\rangle+i|1\rangle|0\rangle)\right]
$$


Where the first ket in each term represents the number of the particles on path $\mathrm{U} 1$ and the second ket represents the number of particles on path U2. It is general the case that any operation on the state of the photon leads to lose and combining vacuum fluctuation. That is the quantum permutation.

The phase is important while modes are distinguished only with their polarization and momentum. In quantum mechanics, states with only a pure phase difference are taken as the same state also. Now that the constituents of the photon and the vacuum fluctuations with phase unknown come together in the same mode to superposition that are the reason the dephase come from.

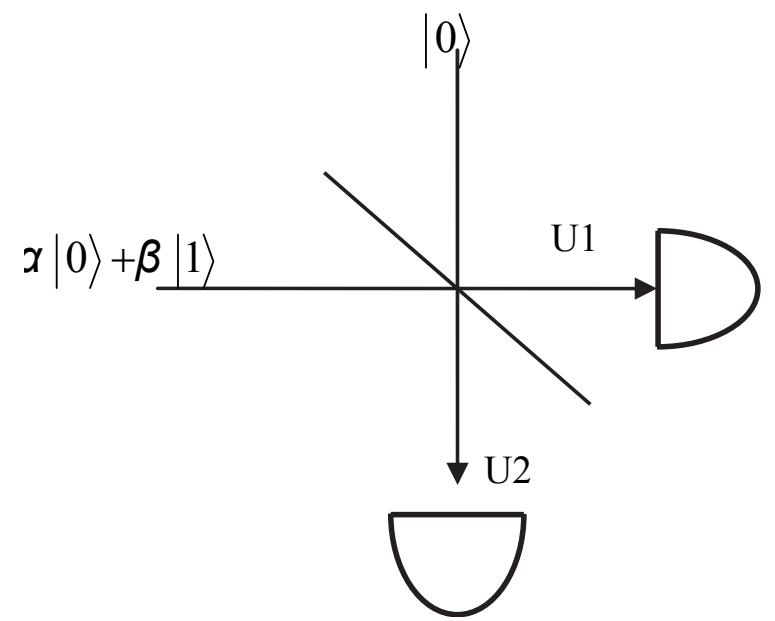

Fig. 11. Single photon is operated by a beam splitter, the vacuum states are involved.

\section{Reconstruction of photons}

Vacuum supports all optical modes and each of the modes can contain constituent of any photon with phase and amplitude arbitrarily defined which are relay on the initial condition. Therefore a photon emitted from a quantum dot or from a single atom will be coupled to all possible modes with equal probability. The interaction of all the emitting mode fields decides the Emitting pattern that has large divergence. The divergent light will soon be scattered to become a part of the fluctuation in the vacuum if it is not immediately collected and focused to a detector and absorbed. Any procedure or operation on the emitted light means mode change and mode structure reorganization.

The actual probability of detecting a photon is decided by the collecting and focusing as much as possible the constituents of the photon. The highest detection efficiency of a photon is always at where the superposition of the mode fields has constructive coherence to the maximum while detection probability of zero may indicate a destructive superposition. Therefore one should consider the mode structure of a photon and the interaction of the mode fields. It is usual to limit the mode number so that can make the modes controllable. It is essential to control the mode selection so that to control the mode structure of a photon. There are two mechanisms to collect energies of the photon. One is the mode competition. The other is control the mode volume geometrically such as in waveguides or in cavities where only limited modes can be excited. 
In case the quantum dot coupled to the surface plasmon in an element of the Yagi-Uda antenna, the emitting energy concentrated in resonant TM mode due to excitation of the surface plasmon so that the TE mode is depressed. This is the resonant enhancement effect. The direction of emitted light by the Yagi-Uda is decided by interaction of all elements composing the antenna. Here only one direction has coherent maximum of superposition.

Reconstructions of photons for quantum information are quite usual. The photons are divided into two or more part and encoded with phase information. On recombining these constituents of the photon, the coherent superposition decides where the maximum detection probability should appear to a detector as predicted. The coherent maximum and the coherent minimum appeared at the same time and in different places showing an entanglement. A successful unitary transformation will guarantee a photon appeared to a detector with probability of 1 while the probability is zero at all other places. However, this transformation can not prevent a detector to sense the vacuum fluctuation.

The vacuum fluctuation has important role in the detection. Since the vacuum contain all possible modes that deserve the ergodic assumption. That is why the quantum permutation with vacuum exists always. In fact, the photon reconstruction is decided eventually under the choice of the detector where the herald mode fields combining necessary energy from the vacuum fields can form a photon the mode structure of which matches the needs for resonant absorption. There has not a photon detector that is quantum state sensitive, for example, a polarization dependent detector. One may consider a detector which is state sensitive so that can decrease the quantum error bits.

In conclusion, photons are different from other particle with static mass. Photons compose by themselves of electromagnetic field modes which are quantized by the electromagnetic interaction. Therefore, mode structure of a photon should be considered so that nonlocality and entanglement of photons could be explained.

\section{References}

Chao, C. Y., \& Kung T.T. (Gong Zutong), (1933). Interaction of hard $\gamma$-ray with atomic nuclei. Nature, 4, (1933) 709

Combes, J. \& Torner, L., (2005). States for phase estimation in quantum interferometry. Journal of Optics B: Quamtum and Semiclassical Optics. 7, (2005) 14-21

Cronstrond, P. \& Jansik, B., (2004). Density functional response theory calculation of threephoton absorption. Journal of Chemical Physics, 121, (2004) 9239-9346

Curto, A. G.; Volpe, G., Taminiau, T.H., et al. (2010). Unidirectional emission of a quantum dot coupled to a nanoantenna. Science, 329, 5994, (20 August 2010) 930-933

Dunningham, J. \& Vedral, V., (2007). Nonlocality of a single particle. Physical Review Letters, 99, (2 November 2007) 180404

Einstein A., (1997). On the quantum theorem of Sommerfeld and Epstein. A translation of the paper appears in "The collection Papers of Albert Einstein", Vol.6, Engel, trans., Princeton U. Press, Princeton, NJ (1997), pp.434, see also: A. Douglas Stone, Physics Today, (August 2005) 37-43

Eiseman, M.D., L. Childress, A., Andre, et al. (2004). Shaping quantum pulses of light via coherent atomic memory. Physical. Review Letters, 93, (2004) 233602

Esteban R., Teperik, T.V., \& Greffet, J. J., (2010). Optical patch antennas for single photon emission using surface plasmon resonances. Physical Review Letters, 104, (15 January 2010) 026802

Frabner, B.,Göppl. M., Fink, J..M., et al. (2008). Resolving vacuum fluctuations in an electrical circuit by measuring the Lamb shift. Science, 322, 5906,(28 November 2008) 1357-1360 
Ghai, D. P., Vyas, S., Senthilkumaran, P., \& Sirohi, R.S., (2009). Vortex lattice generation using interferometric techniques based on lateral shearing. Optics Communications, 282, (2009) 2692-2689

Gisin, N., Ribordy, G., Tittel, W., \& Zbinden, H., (2002). Quantum cryptography. Reviews Modern Physics, 74(1), (January 2002)145-195

Gong Zutong, (1999). Structure theory of photon, PHOTONICS SINICA, 28(1), (1999) 1-10

Greiner W., (2001).Quantum Mechanics, Fouth Ed. Springer-Verlag Berlin Heidelberg, ISBN 7-5062-7263-6/O 530, New York

Hanbury Brown R. \& Twiss, R. Q. (1956). Correlation between photons in two coherent beams of light. Nature (London) 177, (1956) 27-32

Hiskett, Ph. A., Buller, G. S., Loudon, A. Y. et al. (2000). Performance and design of InGaAs/InP photodiodes for single-photon counting at $1.55 \mu \mathrm{m}$. Applied Optics, 39(36), (December 2000) 6818-6829

Hughes,R. J., Morgan, G. I., \& Perterson, C. G., (2000). Quantum key distribution over a 48 km optical fibre network. Journal of Modern Optics 47, 2/3,(2000) 533-547

Kardyna B.E. , Hees, S.S., \& Shields,A.J., (2007). Photon number resolving detector based on a quantum dot field effect transistor. Applied Physics Letters, 90, (2007) 181114

Koashi, M. \& Matsuoka,M., (1996). Photon antibunching by destructive two-photon interference. Physical Review A53, (5), (May 1996) 3621-3624

Kosaka, H., Tomita, A., Namhu, Y., et al. (2003). Single-photon interference experiment over $100 \mathrm{~km}$ for quantum cryptography system using balanced gated-mode photon detector. Electron. Lett. 39(16), (2003) 1199-1201

Mandel, L., \& Wolf, E., (1995). Optical Coherence and Quantum Optics, Cambridge, ISBN 0521 41711 2, New York. pp.476

Maruyama, T., Narusawa, F., Kudo, M., et al. (2002). Development of a near-infrared photon-counting system using an InGaAs avalanche photodiode. Optical Engineering, 41(2), (2002) 395-402

Mason E.J., Albota, M. A., Konig, F., \& Wong, F.N.C., (2002) Efficient generation of tunable photon pairs at 0.8 and $1.6 \mu \mathrm{m}$. Opt. Lett. 27, (2002) 2115-2117

Migdall A.L., Branning, D., Castelletto, S., \& Ware, M., (2002). Single photon source with individualized single photon certifications. Proc. SPIE Vol.4821 (2002) 445-465

Miller A. J. , Sae Woo Nam, and J. M. Martinis, (2003). Demonstration of a low-noise near-infrared photon counter with multiphoton discrimination, Appl. Phys. Lett. 83(4), (2003) 791-793

Mori S., Motoya, M., Namekata, N., \& Inoue, S., (2004). Generation of correlated photon pairs at $1550 \mathrm{~nm}$ by periodically poled Lithium Niobate. Trans IEICE , J87-C(2004)675-685

Muller, A., Herzog, T., Huttner, B. et al. (1997). "plug and play" systems for quantum cryptography. Appl. Phys. Lett. 70(7), (1997) 703-795

Namekata, N., Fujii, G., \& Inoue, S., (2007). Differential phase shift quantum key distribution using single-photon detectors based on a sinusoidally gated InGaAs/InP avalanche photodiode. Appl. Phys. Lett. 91, (2007) 011112

Neves,I., Lima, G., Gomez, A. J. G. et al. (2005). Generation of entangled states of Qbits using twin photons. Phys. Rev. Lett. 94(10), (2005) 100501

Papp, S. B., Choi, K. S., Kimble, H. J. et al. (2009). Characterization of multipartite entanglement for one photon shared among four optical modes, Science, 324, 5928, (2009) 764-771

Pathak A. \& Mandal, S., (2003). Photon-bunching, photon-antibunching and nonclassical photon statistics of coherent light couped to a cubic nonlinear medium. Modern Physics Letters B, 17(5\&6),(2003) 225-233

Pittman T.B., Jacobs, B.C., \& Franson, J.D., (2002). Single photon on pseudodemand from stored parametric down-conversion, Phys. Rev. A 66 (2002)042303 
Rarity, J. G., Ridley, T.E., Ridley, K.D., et al. (2000). Single photon counting for the 1300-1600$\mathrm{nm}$ range by using of Peltier-cooled and passively quenched OnGaAs avalanche photodiodes. Applied Optics, 39(36), (2000) 6746-6753

Roychoudhuri Ch. \& Roy, R., Guist. Ed. (2003). OPN Trends: The nature of light, What is a photon. Optics $\mathcal{E}$ Photonics News, 3(1)

Saleh, B. E. A. \& Teich, M. C.,(Eds.). (1991). Fundamentals of Photonics, John Wiley \& Sons, ISBN 0-471-83965-5, New York, pp.87-88, 249

Santori C., Peton, M., Solomon, G., Dale, Y., et al. (2001). Triggered single photons from a quantum dot. Phys. Rev. Lett. 86, (2001) 1502-1505

Tanzilli S., Riedmatten, H.D., Zbinden, H., et al. (2001). Highly efficient photon pair source using periodically poled lithium niobate waveguide. Electron. Lett. 37 (2001) 26-28

Tomita, A. \& Nakamura, K. (2002). Balanced gated-mode photon detector for quantum-bit discrimination at $1550 \mathrm{~nm}$. Opt. Lett. 27(20), (2002) 1827-1829

Torres, J. P. \&Torner, L. (2005). Quantum state engineering for spatial control of entangled photon pairs. Proceeding of SPIE Vol.5736, (2005) 173-184

Walls D.F., \& Milburn, G.J., (1994). Quantum Optics, Springer-Verlag, ISBN 3-540-58831-0, Berlin Heidelberg, pp.41-58, 199

Walton Z., Sergien, A.V., Atature, M., Saleh, B.E.A., \& Teich. M.C. (2001). Performance of photon-pair quantum key distribution system. J. Mod. Opt. ; 48 (2001) 2055-2063

Wang Jindong., Qin Xiaojuan, Zhang Huani, et al. (2009). A free-space-based differential phase shift quantum key distribution scheme with higher key creation efficiency. Optics communications, 282, (2009) 3379-3381

Wei, G. X., Lu, L-L., Guo, Ch-Sh., (2009). Generation of optical vortex array based on the fractional Talbot effect. Optics Communications, 282, (2009) 2665-2669

Wei Zhengjun, Zhou Peng, Wang Jindong, et al. (2009). An integral gated mode single photon detector at telecom wavelengths. Journal of Physics, D: Applied Physics, 40(22), (2007) 6922-6925

Wootters W.K. \& Zurek, W.H., (1982). A single quantum cannot be cloned, Nature, 299, (1982) 802-803

Yang, Sh., Powers, P. E., \& Zhan, Q., (2009). Experimental verification of focus tailoring using circularly polarized vortex beams with annular pupil mask. Optics Communications, 282, (2009) 4657-4659

Yariv, A., (1988). Quantum Electronics, third edition, John Wiley \& Sons, ISBN 0-471-609978, New York, pp.96-99

Yi, S. W., Lee, S.K., Yang, G.Y. et al. (2004). Three dimensional micro-fabrication using twophoton absorption by femtosecond laser. Procedings of SPIE, Vol.5342, (2004) 137-145,

Yoshizawa, A., Kaji, R., \& Tsuchida, H., (2003). Generation of polarization-entangled photon pairs at $1550 \mathrm{~nm}$ using two PPLN waveguides. Electron. Lett. 39, (2003) 621-622

Yuan, Zhiliang, Beata E. Kardynal, R. M. Stevenson, et al. (2002). Electrically driven singlephoton source. Science, 295,(4 January 2002) 102-105

Yuan, Zh., Wu. Ch., Zhao, H., Zhao, \& Jiang, H., (2005). Imaging of small nanoparticlecontaining objects by finite-element-based photoacoustic tomography. Opt. Lett. 30, (2005) 3054-3056

Zbinden H., Pasel, S., Gisin, N. et al. (2002). Practical quantum key distribution. Quantum Optics In Computing and Communications, Proceedings of SPIE, Vol.4917, (2002) 40-44

Zumuth, S., Ansari, Z., Lepine, E. \& Vrakking, M. J. J. (2005). Single-shot measurement of revival structures in femtosecond laser-induced alignment of molecules. Opt. Lett. 30, (2005) 2326-2328 


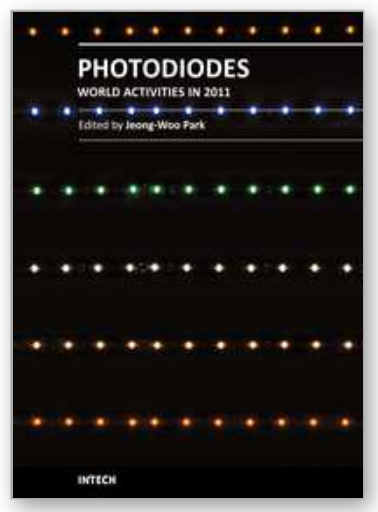

\author{
Photodiodes - World Activities in 2011 \\ Edited by Prof. Jeong Woo Park
}

ISBN 978-953-307-530-3

Hard cover, 400 pages

Publisher InTech

Published online 29, July, 2011

Published in print edition July, 2011

Photodiodes or photodetectors are in one boat with our human race. Efforts of people in related fields are contained in this book. This book would be valuable to those who want to obtain knowledge and inspiration in the related area.

\title{
How to reference
}

In order to correctly reference this scholarly work, feel free to copy and paste the following:

Changjun Liao, Zhengjun Wei and Jindong Wang (2011). Photon Emitting, Absorption and Reconstruction of Photons, Photodiodes - World Activities in 2011, Prof. Jeong Woo Park (Ed.), ISBN: 978-953-307-530-3, InTech, Available from: http://www.intechopen.com/books/photodiodes-world-activities-in-2011/photonemitting-absorption-and-reconstruction-of-photons

\section{INTECH}

open science | open minds

\section{InTech Europe}

University Campus STeP Ri

Slavka Krautzeka 83/A

51000 Rijeka, Croatia

Phone: +385 (51) 770447

Fax: +385 (51) 686166

www.intechopen.com

\section{InTech China}

Unit 405, Office Block, Hotel Equatorial Shanghai

No.65, Yan An Road (West), Shanghai, 200040, China

中国上海市延安西路65号上海国际贵都大饭店办公楼405单元

Phone: +86-21-62489820

Fax: +86-21-62489821 
(C) 2011 The Author(s). Licensee IntechOpen. This chapter is distributed under the terms of the Creative Commons Attribution-NonCommercialShareAlike-3.0 License, which permits use, distribution and reproduction for non-commercial purposes, provided the original is properly cited and derivative works building on this content are distributed under the same license. 